\title{
Kees classification of obstetric and other urine fistulas as based on quantitative and qualitative pelvis tissue loss and on the continence mechanism
}

\author{
KEES WAALDIJK \\ National Obstetric Fistula Center Babbar Ruga, Katsina, Nigeria
}

\begin{abstract}
Objective: A classification of the obstetric and other urine fistulas is presented based on pelvis tissue loss and involvement of the anatomic continence mechanism:
\end{abstract}

Materials and Methods: Kees I fistulas: not involving the continence mechanism;

Kees IIAa fistulas: involving the continence mechanism without (sub)total urethra involvement and without a circumferential defect;

Kees IIAb fistulas: involving the continence mechanism without (sub)total urethra involvement and with a circumferential defect;

Kees IIBa fistulas: involving the continence mechanism with (sub)total urethra involvement and without a circumferential defect;

Kees IIBb fistulas: involving the continence mechanism with (sub)total urethra involvement and with a circumferential defect and miscellaneous;

Kees III fistulas: like ureter fistulas;

Results: The characteristics of each class are described and why they differ from each other by qualitative and quantitative pelvis tissue loss. There is a fluid transition from Kees I into Kees IIAa and from Kees IIAa into Kees IIBa and from Kees IIAa into Kees IIAb and from Kees IIAb into Kees IIBb fistulas. Each fistula class needs to undergo a specific operation:from incision to dissection to repair of pelvis structures, in addition to fistula closure. This is to reconstruct the functional pelvis anatomy.

Conclusion: With this classification it is possible to plan and execute the fistula repair according to the principles of reconstructive surgery and to compare the operation techniques and results in a scientific way. However, these are only guidelines as each fistula constitutes its own unique entity and needs its own customized approach.

Keywords: Obstetric fistula; classification; pelvis tissue loss; endopelvic diaphragm; operation principles

\section{INTRODUCTION}

The variety of fistula caused by obstetric trauma is immense. It ranges from a minute urine fistula with minimal tissue loss, to a cloaca in an empty pelvis with extensive intravaginal lesions and (sub)total loss of the intrapelvic soft tissues, neurologic lesions such as foot drop, extravaginal lesions such as bedsores and loss of labia, urine-induced dermatitis, stones, and systemic lesions such as severe anemia and even cachexia. 
The lesions are caused by intravaginal pressure necrosis, intrapelvic compression of deep structures, immobilization, continuous urine leakage and blood loss. An enormous amount of metabolic energy is consumed during prolonged obstructed labor which may last for days. In addition, fistulas may also develop consequent to practices by the birth attendant such as craniotomy, vacuum delivery, forceps delivery and cesarean section.

In order to make a plan of action to fistula correction, to develop surgical principles, to evaluate different operation techniques and to analyze the results in an objective way, a classification is needed which makes sense; the classification should be objective with clear definitions and parameters and not too complicated.

Based on a retrospective analysis in 775 consecutive patients with fistulas, such a scientific classification was developed and has been used and refined by the author in some 29,000 personal fistula repairs, and in related operations in roughly 25,000 patients during a 35-year period of management of obstetric trauma. The practice involved mainly Nigeria, but also Burkina Faso, Nepal, Niger, Kenya and Tanzania from 1984 up till today. ${ }^{1-7}$

\section{MATERIALS AND METHODS}

\section{Classification}

The rationale of developing this fistula classification method was that since ensuring urine continence after successful closure is the major challenge, any classification must include the urine continence mechanisms as a major decisive inherent component. The anatomic urine continence mechanism in the female consists of the presence of the whole urethra of 3-3.5 $\mathrm{cm}+1-1.5 \mathrm{~cm}$ distal bladder neck; so, in total over $4-5 \mathrm{~cm}$ needs to exist, with the external urethra opening as reference point (Figures 1-4). The classification is presented in Table 1. Figure 4 is a a simplified graphic illustration on a balloon. The classification is based on the progressive quantitative and qualitative amount of pelvis tissue loss and on the progressive involvement of the urine continence mechanism.

In Kees I fistulas there is tissue loss of the bladder, pubocervical musculofascia (PCMF) which is a part of the endopelvic diaphragm (EPD) and anterior vagina wall (AVW) and/or the cervix and/or the uterus. The continence/closing mechanism remains intact. The anchoring of the anterior cervix within the endopelvic diaphragm may be defective. There may be a major tissue loss, and sometimes a trauma to the sacrospinous ligament, (ischio) coccygeus muscles and piriformis muscles. Frequently it is associated with cesarean section (CS) or CS-subtotal hysterectomy or CS-total hysterectomy.

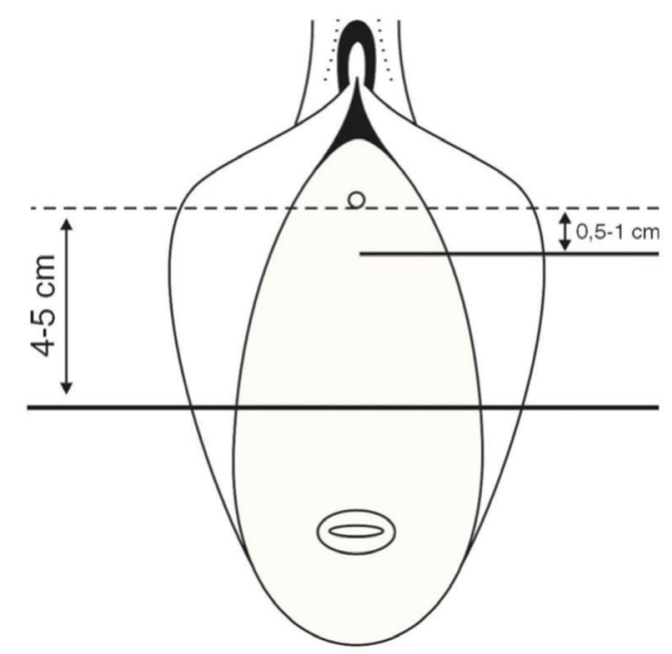

Figure 1. Urine continence mechanism: frontal

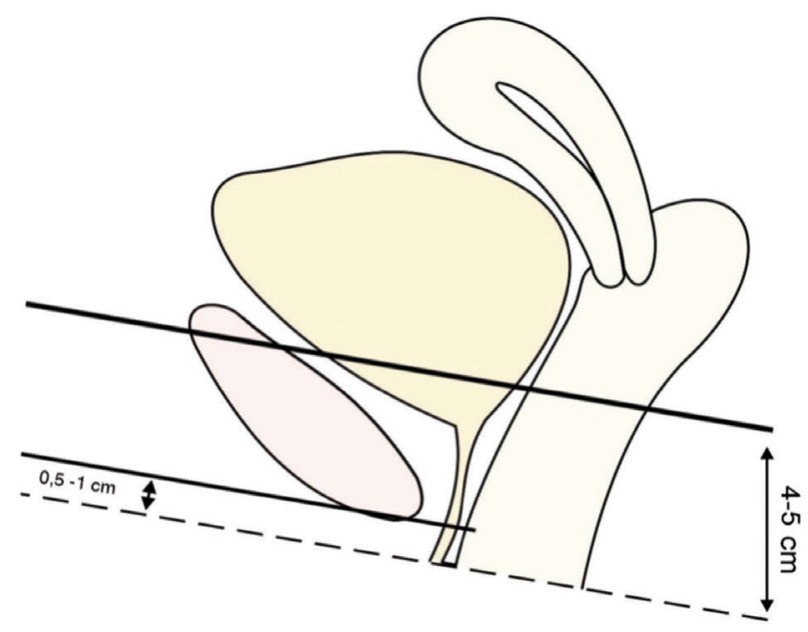

Figure 2. Urine continence mechanism: sagittal

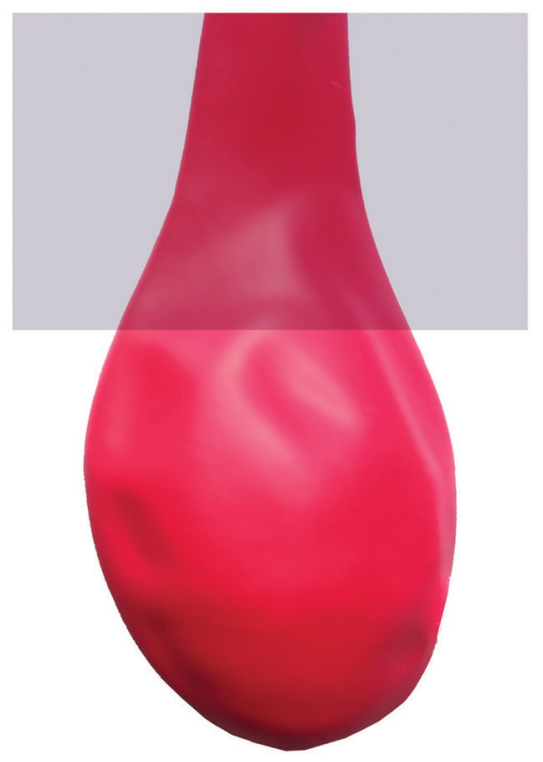

Figure 3. Anatomic urine continence mechanism 


\begin{tabular}{|c|c|c|c|}
\hline $\begin{array}{l}\text { Type of } \\
\text { fistula }\end{array}$ & $\begin{array}{l}\text { Sub- } \\
\text { type }\end{array}$ & Sub-sub-type & Description \\
\hline I & & & $\begin{array}{l}\text { Without involvement of } \\
\text { the continence (closing) } \\
\text { mechanism }\end{array}$ \\
\hline \multirow{6}{*}{ II } & \multirow{3}{*}{ A } & & $\begin{array}{l}\text { Without total or subtotal } \\
\text { involvement of the urethra }\end{array}$ \\
\hline & & $\mathrm{Aa}$ & $\begin{array}{l}\text { Without circumferential } \\
\text { defect }\end{array}$ \\
\hline & & $\mathrm{Ab}$ & With circumferential defect \\
\hline & \multirow{3}{*}{ B } & & $\begin{array}{l}\text { With total or subtotal } \\
\text { involvement of the urethra }\end{array}$ \\
\hline & & $\mathrm{Ba}$ & $\begin{array}{l}\text { Without circumferential } \\
\text { defect }\end{array}$ \\
\hline & & $\mathrm{Bb}$ & With circumferential defect \\
\hline III & & & $\begin{array}{l}\text { Miscellaneous, e.g. } \\
\text { ureter fistulas and other } \\
\text { exceptional fistulas }\end{array}$ \\
\hline
\end{tabular}

In Kees IIAa fistulas there is tissue loss of the bladder, urethrovesical (UV)-junction/trigonal ring, detrusor loops, proximal and possibly mid urethra, PCMF/EPD and AVW (with the cervix and/or the uterus). In addition, minor to moderate involvement of the continence/closing mechanism exists. There may be a slight trauma to the ATF, arcus tendineus of the levator ani muscle (ATLAM) and the levator ani musculature. There may be a transverse or a quartercircular defect within the PCMF/ EPD. This may lead to indirect disruption or weakening of its connection to the paraurethral pubis bones and paraurethra ATF. The ATF and the ATLAM are usually intact. The defect within the PCMF/EPD is larger than the fistula. The fistula may be found within this defect.

In Kees IIAb fistulas there is a circumferential tissue loss of the bladder neck, UV-junction and trigonal ring, detrusor loops, proximal or mid urethra and tissue loss of posterior pubourethral ligaments, PCMF/EPD, AVW (and cervix and/or uterus), ATF, ATLAM, pubococcygeus muscles and iliococcygeus muscles. In addition, a trauma to the obturator internus muscles, obturator membrane and coccygeus muscles may occur, with eventual loss of the pubis bone periost and the pubis symphysis cartilage, with moderate to major involvement of the continence/closing mechanism. There is no functional tissue connection whatsoever between the traumatized urethra or what is left of it, and the traumatized bladder neck. The urethra has usually been retracted distally, and the bladder proximally in opposite directions whilst the paravesical space is opened. The stabilizing support of the PCMF/EPD needed for the physiologic urethra continence/closing function, is usually lost and there is no connection between what is left of the traumatized PCMF/ EPD and the paraurethral pubis bones. The anterior part of the PCMF/EPD, together with the paraurethral and distal part of the ATF are completely lost.

In Kees IIBa fistulas there is major tissue loss of the urethra and of the UV-junction/trigonal ring, detrusor loops, bladder, PCMF/ EPD and AVW (and cervix and/or uterus) with major involvement of the continence/closing mechanism. Though there is tissue loss of the anterior part of PCMF/EPD, the ATF, ATLAM and levator ani musculature are intact. Due to the natural tissue forces inside the human body, the endopelvic diaphragm with adherent traumatized posterior bladder neck retracts towards the cervix/ sacrum. There is also a dislocation and/or loss of the posterior UV-junction. The stabilizing support of the PCMF/EPD needed for the physiologic urethra continence/closing function has been lost. This is because of an anterior defect in the PCMF/EPD whilst its anterior connection to the immediate paraurethral pubis bones has been disrupted.

In Kees IIBb fistulas there is total or subtotal circumferential tissue loss of the urethra, UV-junction/trigonal ring, bladder neck, detrusor loops and tissue loss of the posterior pubourethral ligaments, PCMF/EPD, AVW (and cervix and/or uterus), ATF, ATLAM, pubococcygeus/iliococcygeus muscles. In addition, there may also be a trauma to the obturator internus muscles, obturator membrane and coccygeus muscles with eventual loss of pubis bone periost and pubis symphysis cartilage. There is an extensive involvement of the continence/closing mechanism whilst the paravesical space is opened. There is no functional tissue connection whatsoever between what is left of the severely traumatized urethra if anything is left at all of it, and the traumatized bladder neck, whilst the bladder has retracted proximally. The retraction is limited anteriorly by the loose fixation of the anterior bladder onto the posterior symphysis and anterior abdominal wall. The anterior part of the PCMF/ EPD has been lost completely together, with bilateral loss of the paraurethral and distal part of the ATF. In addition, its bilateral semicircular anterior connection onto the paraurethral pubis bones and bilateral ATF is directly disrupted. In extensive trauma there may even be a complete bilateral loss of the ATF from the paraurethra and up to the ischium spine. The cephalad part of the pubococcygeus muscle has been lost with total or subtotal loss of the paraurethral ATLAM. In extensive trauma, the cephalad part of the iliococcygeus muscle is lost as well, with complete ATLAM loss from the paraurethra up to the ischium spine. this is associated with a very extensive tissue loss, making the surgical management very complicated in these fistulas. Frequently an empty pelvis is found with bare pubis bones. In these cases, the fistula may be inoperable. 


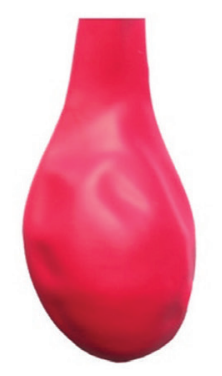

bladder + urethra

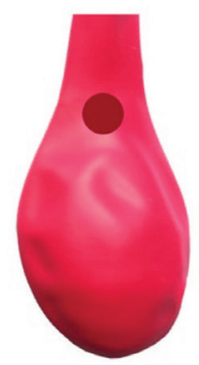

kees IIAa

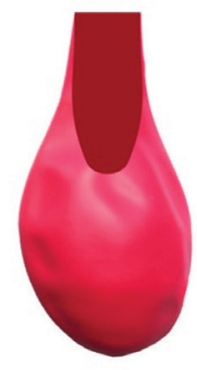

kees IIBa

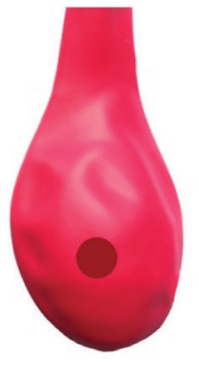

kees I

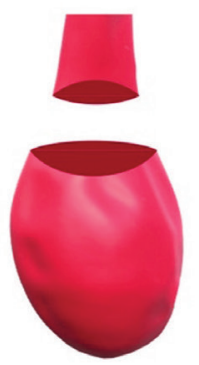

kees $\| \mathrm{Ab}$

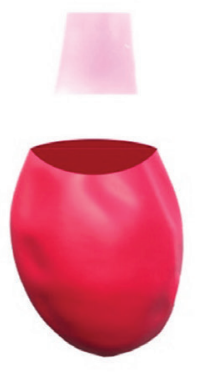

kees $I \mathrm{BB}$
Figure 4. Kees urine fistula classification

The transition from Kees I into Kees II fistulas is at $4-5 \mathrm{~cm}$ and the transition from Kees IIA into Kees IIB fistulas is at 0.5-1 cm from the external urethra opening.

The Kees III fistula is an independent class, including a ureter fistula, a fistula between the bladder and bowel, and fistula between the bladder and skin.

\section{Mechanism of action in circumferential fistulas}

Due to the total disruption of the bilateral anterior PCMF/EPD from the pelvis wall, and the circumferential disruption of the bladder neck from the urethra, the PCMF/EPD and bladder neck retract towards the cervix/sacrum. This retraction is restricted by the loose fixation of the anterior bladder neck to the posterior symphysis and anterior abdominal wall by connective tissue. Since the bilateral semicircular anterior fixation of the PCMF/EPD to the ATF and paraurethral pubis bone is also disrupted, the loose PCMF/EPD with adherent posterior bladder wall retracts towards the anterior bladder wall. This is due to the natural tissue forces. The bladder then loses its saucer shape configuration when empty. The PCMF/EPD with adherent posterior bladder wall move anteriorly, towards the symphysis, and cephalad towards the superior pubis bone rami. They may then reconnect with the obturator internus fascia or superior pubis bone rami, at a more cephalad level resulting in deep bilateral anterior vagina sulci. This may extend up to the superior pubis bone rami. This mechanism explains why the fistula retracts and becomes less accessible behind the pubis symphysis. In some instances the anterior bladder wall retracts cephalad above the superior brim of the symphysis. It is the cephalad part of the levator ani muscle, together with its origin ATLAM, which are lost whilst the caudad part with its insertion into the levator plate and coccyx is still intact. If there is a major loss of the levator ani musculature, the result is an empty pelvis with bare pubis bones. This consequence is always combined with total or subtotal loss of the ATF and ATLAM from the paraurethra up to the ischium spine.

\section{Operative principles}

Since the fistula is a part of an obstetric trauma, the functional pelvis anatomy has to be reconstructed, while the fistula is closed during the procedure. Restoring the anatomy will ensure resuming the normal physiology. Special attention should be paid not to occlude the ureters. The surgical procedures may vary according to the present lesions and characteristics, but always include incision, restoring the bladder and urethra closure, and PCMF/EPD reconstruction and anterior vagina wall adaptation..

In Kees I fistulas, the incision extends around the fistula's edge with eventual bilateral transverse extension. Alternatively, a "physiologic" incision should be carried out at the anterior cervix in vesicocervical and vesicouterine fistulas. Sharp dissection and transverse, oblique or longitudinal bladder closure as needed by a single layer of inverting absorbable sutures. The surgeon then needs to check the connection of PCMF/EPD onto the anterior cervix and correct it if needed. Then checks on the closure and continence. Finally, insertion and fixation of catheter should be followed by AVW/cervix adaptation.

In Kees IIAa fistulas, physiologic incision is carried out within the vagina rugae through the fistula and then around the fistula edge. Then a transverse repair of the defect within the PCMF/EPD, with bladder or urethra closure using a single layer. During the procedure the surgeon should check on any loose connection of PCMF/EPD to bilateral anterior pelvis wall and correct this if necessary. and check the closure and continence. Then the surgeon should insert and fixate the catheter and make sure there is a transverse AVW adaptation rarely, a longitudinal closure is indicated. 
In Kees IIAb fistulas, the first incision should start around the fistula's edge. The second physiologic incision should be carried out through the fistula in between the anterior bladder neck and the posterior symphysis, or the other way around, for circumferential dissection and mobilization of the bladder neck, distal advancement of the bladder neck and circumferential end-to-end anastomosis as vesicourethrostomy. Then a quartercircular fixation of the PCMF/EPD to the paraurethral pubis bones and bilateral ATF should be made. At this stage the surgeon should check on the closure and continence, insert and fixate the catheter and then AVW adaptation. If a complete circumferential end-to-end anastomosis is not possible or too complicated, a 4/5-, 3/4- or 2-/3 circumferential dissection is performed followed by 4/5-, 3/4- or 2/3- "end-toend" vesicourethrostomy, where the symphysis "closes" the gap between the anterior bladder neck and the anterior urethra.

In Kees IIBa fistulas, wide $\mathrm{H}$ incision should be carried out bilaterally, from the urethral fistula through the sulci with the horizontal part at the proximal fistula edge. This should be followed by sharp dissection of the AVW, mobilization of the retracted paraurethral tissue, and then by a longitudinal inverted $T$ urethral reconstruction with repositioning of dislocated posterior UV-junction. The next stage is refixation of the PCMF/EPD onto the paraurethral pubic bones. The surgeon then should check on closure and continence, insert and fixate the catheter and reconstruct the AVW using the already dissected AVW as an advancement flap.

In Kees IIBb fistulas, the author prefers to use a two-stage procedure. Initially, First stage: Start with a wide $\mathrm{H}$ incision bilaterally from the "urethra" through the sulci. The horizontal part should be at the proximal fistula edge. Then, a second dissecting incision should be carried out, between the anterior bladder neck and the posterior symphysis, for circumferential dissection/mobilization, distal advancement of mobilized bladder (neck) and tapering fixation into the original "external urethra opening". Then a semicircular anterobilateral fixation of PCMF/EPD onto paraurethral pubis bones and bilateral "ATF" should be performed and then AVW adaptation. The surgeon should then check the continence, insert and fixate the catheter. Ifa post-repair incontinence develops, a second stage urethral reconstruction should be performed using bladder tissue as described under repair of Kees IIba.

A one-stage operation using an anterior, lateral or posterior bladder flap for urethra reconstruction is possible, but the results are not optimal.

The Kees III fistulas need a customized approach, e.g. abdominal or vaginal ureter re-implantation

\section{RESULTS}

\section{Outcome of fistula surgery}

The series include 1,716 consecutively operated patients, who had a fistula for less than three months and were not operated before. A final follow up examination was performed 5-6 months postoperatively. The results are shown in table II. All patients were operated by one surgeon, the author, under the same pre, intra- and post-operative conditions. The same team and the same instructions were used. The table shows that the more the continence mechanism is affected, the more post-repair incontinence might develop. Kees IIBb fistulas which were the most complicated to repair, resulted with the worst outcome of healing and continence.

\begin{tabular}{|c|c|c|c|c|}
\hline $\begin{array}{l}\text { Type/ } \\
\text { subtype }\end{array}$ & $\begin{array}{l}\text { Number of } \\
\text { patients }\end{array}$ & $\begin{array}{l}\text { Healed after } \\
1^{\text {st }} \text { surgery } \\
n,(\%)\end{array}$ & \begin{tabular}{|l|} 
Final \\
healing - \\
$\mathrm{n},(\%)$
\end{tabular} & $\begin{array}{l}\text { Still } \\
\text { incontinent } \\
\mathrm{n},(\%)\end{array}$ \\
\hline 1 & 243 & $238(97.9)$ & 242 (99.6) & $1(0.4)$ \\
\hline IIAa & 888 & 868 (97.4) & $888(100)$ & $11(1.2)$ \\
\hline IIAb & 366 & $333(91.0)$ & 353 (96.4) & $30(8.5)$ \\
\hline $\mathrm{IIBa}$ & 87 & 80 (96.4) & 86 (98.9) & $14(16.3)$ \\
\hline $\mathrm{IIBb}$ & 132 & 114 (86.4) & $121(91.7)$ & $59(48.8)$ \\
\hline
\end{tabular}

\section{DISCUSSION}

The variety of the complex obstetric trauma is immense, so that it is impossible to develop an ideal classification of the obstetric fistula. In fact, each fistula constitutes of its own specific entity. Therefore, within each fistula type, many subtypes exist. Not only the fistula has to be classified, but all the lesions and defects have to be objectively documented in writing to be completely transparent. Vaginal strictures, scarring, stenosis, fistula size and previous repair attempts are not a part of the classification, but they may complicate the operation and decrease healing and continence success.

The characteristic of the circumferential fistulas is the complete disruption of what is left of the traumatized urethra, from the traumatized bladder neck, with opening of the paravesical space. The classification should be performed during examination under anesthesia at the beginning of the reconstructive surgery, with the patient totally relaxed and in the exaggerated lithotomy position after a thorough examination of all the obstetric trauma lesions that exist. Only then, a plan of action should be made. 


\section{CONCLUSION}

With the Kees' classification it is possible to plan and execute a fistula repair according to the principles of reconstructive surgery, and to compare the operation techniques and results in a scientific way. However, since the variety of fistula variations is immense and there are no sharp demarcations transition between the different types is possible. Therefore this classification should be used as a guideline. Each fistula needs its own customized approach, and that is exactly what makes obstetric fistula surgery so intriguing!

\section{Ethics}

Ethics Committee Approval: For this study type an ethics committee is not necessary.

Informed Consent: Not necessary.

Peer-review: Externally peer-reviewed.

\section{DISCLOSURES}

Financial Disclosure: The author declared that this study received no financial support.

\section{REFERENCES}

1. Hafferl A. Lehrbuch der topographischen Anatomie. Springer Verlag; 1957.

2. Marani E, Koch WFRM. The pelvis: structure, gender and society. Springer Verlag; 2014.

3. Petros PE. The female pelvis floor, Function Dysfunction and Management according to the Integral Theory. Springer Heidelberg; 2010.

4. Waaldijk K: The (surgical) management of bladder fistula in 775 women in Northern Nigeria. PhD thesis Univ. of Utrecht; 1989.

5. Waaldijk K: Obstetric trauma surgery; art and science: 004 basics. Printmarkt; 2008.

6. Waaldijk K: Obstetric trauma surgery; art and science: 013 corpus intrapelvinum with endopelvic diaphragm. Printmarkt; 018.

7. Waaldijk K: Obstetric trauma surgery; art and science: 020 Kees urine fistula classification. Printmarkt; 2020. 